\title{
Estrategias de sobrevivencia educativa de los egresados de bachillerato tecnológico de CECyTEJ, El Salto
}

\author{
Strategies for the educational survival of alumni of the \\ technological baccalaureate program of CECYTEJ EI Salto, \\ Jalisco, Mexico
}

DOI: https://doi.org/10.32870/dse.v0i24.1065

\section{Christian López Ochoa*}

Resumen

Este artículo se presenta como propuesta para pensar que es posible atender el fenómeno del abandono escolar si se comprenden los recursos que despliegan los jóvenes que permanecen estudiando en entornos con problemáticas sociales. Para ello, se utiliza el concepto Estrategias de Sobrevivencia Educativa, el cual sirve para analizar las experiencias y trayectorias escolares de egresados de bachillerato tecnológico que estudiaron en el Colegio de Estudios Científicos y Tecnológicos del Estado de Jalisco (CECyTEJ), ubicado en El Salto, municipio del Área Metropolitana de Guadalajara, donde se identificó que los jóvenes sortean problemas para concluir dicho nivel educativo. La investigación se llevó a cabo con un enfoque sociocultural y el método biográfico para recuperar la voz de los sujetos de estudio. Se utilizó como perspectiva teórica el análisis institucional para comprender las producciones culturales que los sujetos llevan a cabo en su paso por la institución educativa.

Palabras clave: jóvenes - bachillerato tecnológico - trayectorias educativas - familia y escuela.

\begin{abstract}
This article presents a proposal to address the high school dropout phenomenon by understanding the resources used by young people who continue studying in environments with social problems. We used the concept of Educational Survival Strategies to analyze the experiences and school trajectories of graduates of the technological baccalaureate program of Colegio de Estudios Científicos y Tecnológicos del Estado de Jalisco (CECYTEJ) in El Salto, a municipality in the metropolitan area of Guadalajara where we identified that young people have problems to complete that educational level. Our research focused on the sociocultural aspect, using the biographical method to retrieve the voice of our study subjects and institutional analysis as a theoretical approach to understand our subjects' cultural productions that the subjects implement in their passage through the educational institution.
\end{abstract}

Keywords: youth - technological baccalaureate - educational trajectories - family and school.

* Doctor en Educación. Líneas de investigación: Jóvenes y bachillerato; trayectorias educativas y laborales; estudios institucionales; estudios socioculturales sobre educación. Benemérita y Centenaria Escuela Normal de Jalisco. chlooch@hotmail.com 


\section{Introducción}

En este trabajo se recuperan algunos resultados de la investigación que realicé como estudiante del Doctorado en Educación en la Universidad de Guadalajara y que tuvo por objetivo conocer cómo se constituyen las trayectorias educativas y laborales de los egresados de bachillerato tecnológico del municipio de El Salto, Jalisco, con el fin de comprender el impacto que tiene el corredor industrial que ahí se ubica en la oferta educativa disponible para los jóvenes.

El fragmento que aquí se presenta se enfoca en recuperar las estrategias que los egresados de bachillerato tecnológico del CECyTEJ, El Salto, emplearon para permanecer matriculados en esa institución y no abandonarla, ya que a través de sus testimonios se identificó que, tanto en ese municipio como en el plantel, el abandono escolar se presentaba como un fenómeno persistente entre generaciones, como se muestra en la tabla 1. Tales estrategias se consideran formas de resistencia y sobrevivencia educativa, pues permiten observar las diferentes acciones, razonadas e intencionales, que los jóvenes emprenden para permanecer afiliados a la institución educativa, en un entorno donde el rezago económico de las familias dificulta el tránsito de los hijos a niveles educativos superiores.

Tabla 1. Incidencia de interrupción y abandono escolar intergeneracional

\begin{tabular}{|c|c|c|c|}
\hline Nombre & Compañeros al ingresar & $\begin{array}{l}\text { Compañeros al fina- } \\
\text { lizar }\end{array}$ & Año de egreso \\
\hline Socorro & 50 & 30 & 2006 \\
\hline El Federal & 50 & 25 & 2006 \\
\hline La Comunicadora & 38 & 33 & 2007 \\
\hline El Freelance & 55 & 25 & 2007 \\
\hline Gaby & 30 & 25 & 2010 \\
\hline Alan & 50 & 25 & 2013 \\
\hline La Doña & 48 & 20 & 2015 \\
\hline La Deportista & 45 & 30 & 2015 \\
\hline Grande & 40 & 20 & 2015 \\
\hline Santos & 40 & 30 & 2015 \\
\hline Karla & 55 & 20 & 2015 \\
\hline Larrión & 50 & 30 & 2015 \\
\hline Hilda & 45 & 22 & 2015 \\
\hline Manuel & 45 & 35 & 2016 \\
\hline La Maestra & 41 & 18 & 2016 \\
\hline María & 45 & 30 & 2016 \\
\hline
\end{tabular}

Fuente: Elaboración propia con información recabada de los egresados.

El enfoque de la investigación es sociocultural y se apoya en el análisis institucional como referencia teórica (Fernández, 1994; Remedi, 2004), pues éste considera un doble análisis: el 
de la realidad externa al sujeto y el de la realidad que lo constituye. Esto contribuye a generar conocimiento acerca de lo que el sujeto produce a partir de su inmersión en una cultura institucional, en este caso, se trabajó para comprender la internalización de la cultura de la educación tecnológica que llevan a cabo los egresados.

El diseño del instrumento metodológico se inspiró en los "relatos de vida" que Daniel Bertaux (2005) propone para la reconstrucción de la historia vivida por los sujetos, y que parte de una postura objetivista centrada en el entendimiento de su mundo social, actividad u oficio; en este caso, del que constituyen los egresados de bachillerato tecnológico en el municipio de El Salto. Bertaux puntualiza que el relato de vida tiene una triple función. La primera es de exploración, para conocer sobre el objeto social que se estudia. La segunda es analítica, donde el investigador ya cuenta con mayores referencias sobre el objeto de estudio, y establece nuevas hipótesis que irá explorando. La tercera función es expresiva, pues al estructurar el relato y convertirlo en testimonio, también se ilustra sobre lo que viven los sujetos. Se considera entonces que al hacer esto, la investigación dinamiza su función de comunicar.

Para ello, se realizaron entrevistas biográficas con diferentes personas que entonces laboraban o habían laborado en el plantel, así como con 16 egresados pertenecientes a distintas generaciones durante un periodo de diez años, de 2006 a 2016, una temporalidad que se considera representativa, pues el plantel se fundó en 1998. Se empleó la técnica conocida como Bola de nieve para la recolección de datos, con el fin de que cada uno de ellos sugiriera a otros informantes. Los encuentros se dieron en los hogares de algunos egresados o en espacios del municipio que ellos mismos sugirieron. Posteriormente se transcribieron las entrevistas y se analizó la información de manera colectiva, tratando de encontrar aspectos comunes en sus experiencias, lo que permitió identificar el tema que aquí se presenta.

Vale la pena mencionar que a lo largo del texto se identifica a los egresados con pseudónimos que hacen referencia al apodo que recibieron de sus compañeros durante el bachillerato, mientras que a otros se les nombra con apelativos que tienen que ver con la ocupación que desempeñaban al momento de la entrevista.

Para indagar en la configuración de sus trayectorias educativas se emplearon dos categorías de análisis: en la primera se analiza el papel que desempeña el bachillerato tecnológico en la afiliación y permanencia de los jóvenes a la institución educativa, así como de la labor que ésta desarrolla como espacio de transmisión cultural que los involucra con el mercado de trabajo de la industria local. En la segunda categoría se analizan las experiencias que los sujetos tuvieron mientras fueron estudiantes de bachillerato, haciendo hincapié en la influencia que recibieron de sus familias en la constitución de sus trayectorias, pues la familia se presenta como la primera institución a la que se está afiliado, y en la mayoría de los casos es la proveedora principal de recursos materiales y simbólicos para que los jóvenes estudien, decisión que permitió distinguir algunas diferencias en las historias de los entrevistados, aún y cuando estos se ubicaban en un estrato social más o menos uniforme. 


\section{Los estudios sobre trayectorias educativas y el abandono escolar}

Los estudios sobre abandono escolar son recurrentes en México ya que es uno de los problemas persistentes en el sistema educativo nacional. La Secretaría de Educación Pública (SEP) ha señalado que este fenómeno se recrudece en la Educación Media Superior (EMS), nivel al que se incorpora $70 \%$ de los jóvenes entre 15 y 17 años de edad. Asimismo, se estima que sólo siete de cada 10 jóvenes que ingresan terminan el periodo de estudios (INEE, 2018).

Imagen 1. Evolución a nivel nacional del abandono escolar en bachillerato

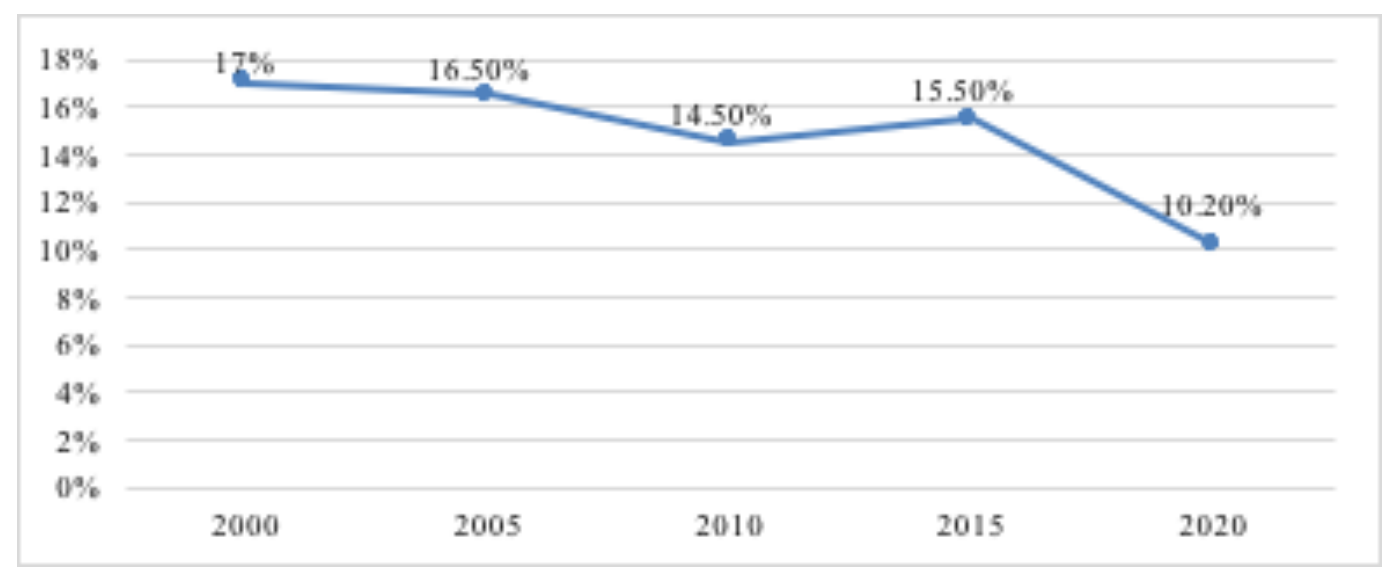

Fuente: Cálculo de datos del INEE (2017), SEMS (2015) y SNIE (2021).

En el aspecto académico, diferentes investigadores han estudiado las trayectorias escolares de los jóvenes en México para comprender las causas que abonan al abandono escolar. Al respecto, Blanco, Solís y Robles (2015) Ilevaron a cabo la Encuesta sobre Trayectorias Educativas y Laborales 2010 (ETEL) con personas que tenían entre 18 y 29 años de edad. Identificaron que siguen siendo los jóvenes de estratos socioeconómicos bajos los más propensos a problemas como la interrupción y el abandono escolar.

Estos autores mencionan que la mayoría de los factores registrados están asociados al peso que tiene la familia de origen en la trayectoria escolar, como son: el número de hijos que hay en el hogar, la falta de alguno de los padres y la no-corresidencia con ellos. Estos factores se suman a los que ya habían reportado otros investigadores, como son: el origen social, el turno en que se estudia, el sostenimiento del plantel al que se asiste y el inicio de la trayectoria laboral a edad temprana, entre otros (Boado, 2013; De la Rocha, 2006; Enríquez, 2014; Giorguli, 2002).

Por su parte, Saraví (2016) realizó un estudio sociocultural en el que comparó la experiencia escolar de jóvenes que llevaron a cabo sus trayectorias en bachilleratos de sostenimiento público y privado. En sus hallazgos reportó que existen diferencias marcadas en ambos grupos de estudiantes ya que los matriculados en escuelas particulares suelen experimentar lo que de- 
nomina como "escuela total", una experiencia escolar más lineal y previsible, sin interrupciones marcadas, pues no suelen vincular lo educativo con lo laboral, debido al respaldo familiar con el que cuentan.

En contraste, señala que algunos estudiantes de bachilleratos públicos que se encuentran en zonas populares, tienen una experiencia escolar "acotada" pues provienen de familias que no pueden apoyarlos en lo educativo y sobrellevan situaciones que terminan por quitarle centralidad a la escuela, como es el empleo a edad temprana, incorporarse a grupos saturados donde encuentran profesores rebasados por la demanda de alumnos y por las carencias en infraestructura educativa.

A nivel internacional, existen referentes que permiten pensar en el rol que desempeña la familia en la constitución de las trayectorias escolares, como son las investigaciones que Bourdieu realizó con estudiantes y que se pueden consultar en libros como La distinción (1998) y Los herederos (2004), donde el autor analiza el logro escolar de los jóvenes que provienen de diferentes clases sociales y que están matriculados en el sistema educativo francés. En esas obras, Bourdieu observa las formas de distinción que se generan a partir de la acumulación de capital económico, lo que diferencía la posesión de recursos materiales con los que se presentan los estudiantes a la escuela. También señala que existe una distinción inmaterial o simbólica, marcada por el acceso a otros tipos de capitales como el cultural, que transmiten o heredan los padres a sus hijos al darle centralidad a lo educativo, fomentando el acceso a formas y bienes culturales para enriquecer su acervo, como pueden ser viajes, libros, cine u otras formas de la denominada "alta cultura"; o el capital social, bajo el supuesto de que las escuelas se constituyen como espacios para el establecimiento de relaciones que posibilitan un mejor acceso hacia el mundo laboral.

La familia ocupa un lugar importante en el análisis institucional, ya que las instituciones tienen la potencia para estructurar la biografía de los sujetos a través de la internalización de la cultura institucional a la que se está adscrito. Una de las instituciones con mayor peso en los primeros años de vida es la familia, ya que "la autoridad parental ejerce normas expresas para el moldeamiento del comportamiento del sujeto" (Fernández, 2004: 17).

El estudio de las trayectorias educativas abona a la exploración sobre este fenómeno, pues implica la sistematización de las distintas etapas que vive el individuo "durante y después de finalizar su formación en una determinada profesión o al insertarse en una actividad laboral u oficio, en cuanto a lo profesional, laboral, económico y social" (Jiménez, 2009: 2); también contribuyen a recuperar su conocimiento sobre las situaciones que se presentan en determinados contextos, así como las acciones que realizan las personas para sobreponerse a ellas.

\section{Los jóvenes y el bachillerato tecnológico en El Salto}

Esta investigación se llevó a cabo en el municipio de El Salto, debido a que ha experimentado con fuerza el impacto de la modernidad. Durante el siglo XX pasó de ser una población enfoca- 
da en la agricultura a convertirse en una comunidad industrializada, donde se han establecido algunas de las empresas nacionales e internacionales que tienen mayor presencia económica en el país.

Con los años se convirtió en uno de los municipios que hospedan a un importante número de empresas nacionales y extranjeras, principalmente a las centradas en el campo de la electrónica, sobre el que diversos investigadores han señalado que se experimentan condiciones de flexibilización laboral que limitan los derechos fundamentales de sus trabajadores, como son la salud, la alimentación y el acceso a la vivienda' (Casillas, 2013; CEREAL, 2015; Delgado, 2017; Gabayet, 2006; Ramos, 2014).

Otra de las problemáticas que padece el municipio, también relacionada con la industrialización, es la contaminación del que alguna vez fue su principal recurso natural, el Río Santiago. La contaminación del río ha motivado que la sociedad civil demande soluciones para su saneamiento, pues investigadores y organismos han evidenciado cómo la industrialización del municipio no ha procurado un modelo de crecimiento sustentable, lo que genera daños en la salud de los pobladores (Greenpeace, 2012; McCulligh, 2006; Rodríguez, Cota 2006; Partida, 2002; Celis, 2010; Regalado, 2015).

Aspectos como el educativo también parecen tener relación con la industrialización del municipio, pues el bachillerato tecnológico es el nivel más alto al que los jóvenes pueden aspirar sin tener que migrar o llevar a cabo largos recorridos hacia otros municipios. Se pudo observar que en el caso del plantel del CECyTEJ localizado en la zona, la oferta educativa se dirige a preparar a los jóvenes para que, una vez egresados, se incorporen a la industria de la electrónica al ofrecer carreras como: Técnico en informática, Técnico en programación, Técnico en mantenimiento y equipos de cómputo, Técnico en electrónica y técnico en seguridad industrial y protección civil.

El bachillerato tecnológico es una de las tres grandes modalidades que integra el Sistema Nacional de Educación Media Superior. ${ }^{2}$ En ella, los estudiantes cursan al mismo tiempo materias de bachillerato general y carreras técnicas que eligen o les son asignadas, por lo que una vez egresados obtienen una certificación con la que pueden optar entre incorporarse al mercado de trabajo como técnicos o seguir estudiando en una institución de educación superior.

Al realizar esta investigación (2016-2019) se observó que la oferta de educación media superior en el municipio resulta relativamente limitada, pues existían tres planteles a los que los jóvenes podían inscribirse; dos de ellos de educación tecnológica, uno perteneciente al sistema CONALEP ${ }^{3}$ y otro al CECyTEJ, mientras que el plantel restante era un de bachillerato general coordinado la Universidad de Guadalajara (UdeG). Se eligió tomar al CECyTEJ como parte del

1 El CEREAL (2015), informó que en el Corredor industrial de El Salto hay cuatro plantas de las 10 empresas más importantes de electrónica a nivel mundial.

2 La educación media superior en México se organiza en tres modalidades: bachillerato general, bachillerato tecnológico y profesional técnico. 3 Colegio Nacional de Educación Profesional Técnica. 
objeto de estudio, pues era la institución con más bachilleratos tecnológicos en el estado (26 planteles en 21 municipios), y porque para entonces contaba con más alumnos que CONALEP en el municipio.

La reconstrucción de las trayectorias educativas de los egresados permitió conocer los aspectos socioculturales que han constituido sus experiencias escolares, como son: el acompañamiento familiar que recibieron; los programas que cursaron; las condiciones que encontraron en el municipio para estudiar el bachillerato y las acciones que llevaron a cabo para egresar. El análisis de estos aspectos permitió indagar en el sentido que los egresados producen acerca de lo educativo, el impacto que la orientación tecnológica tiene en el entorno que habitan y sobre las estrategias individuales y colectivas que desarrollan para permanecer matriculados en un entorno educativo que se puede considerar constreñido, pues resulta insuficiente para cubrir la demanda de los jóvenes que cada año buscan incorporarse al bachillerato.

Para responder a estas inquietudes e indagar en los recursos que los estudiantes de bachillerato de El Salto experimentan para permanecer matriculados, se propone pensar que cada uno de ellos empleó estrategias que les permitieron sobrevivir a lo educativo. Se consideran estrategias de sobrevivencia pues se identificó que con frecuencia los jóvenes ven interrumpidas sus trayectorias escolares al llegar a este nivel educativo, por lo que indagar en las experiencias de los egresados permite conocer los recursos materiales e inmateriales en los que se apoyan y cómo es que se abren paso a través de un sistema educativo que para algunos resulta limitado y en ocasiones excluyente. ${ }^{4}$

A los egresados con los que se dialogó se les considera estrategas de sus trayectorias educativas, pues casi todos se hicieron cargo de éstas desde temprana edad, por lo que este trabajo permite indagar en la capacidad de actoría que los jóvenes desarrollan para permanecer estudiando.

Diferentes estudios sociológicos han abonado a este tema y permiten profundizar en las particularidades que los sujetos experimentan en sus entornos. Entre ellos, destacan los que realizó Bourdieu para analizar el desarrollo de los campos sociales (2001). También se encuentran los de Dubet $(2005,2010)$, sobre la construcción de la experiencia de los estudiantes y la manera en que estos significan su paso por las instituciones educativas. En esos trabajos se analiza cómo los sujetos desarrollan estrategias para moverse en el interior de las instituciones, asumiendo que sus acciones implican respuestas racionales y estratégicas para enfrentar las condiciones estructurales que se les presentan. La capacidad de desarrollar esas estrategias es resultado de su capacidad de actoría, traducida como la capacidad de llevar a cabo acciones a partir de procesos reflexivos que permiten al sujeto resolver las situaciones que se presentan en el medio en el que se desenvuelve (Giddens, 2011).

4 En el ciclo escolar 2016-2017 se informó que en Jalisco se tenía una cobertura en educación media superior de $72.3 \%$. Fuente: Indicadores. sej.gob.mx 
El concepto de experiencia que aquí se emplea recupera la propuesta de Dubet (2005, 2010), quien lo utiliza para analizar la constitución del "ser estudiante" y los retos que eso implica, las situaciones que se experimentan y los sentidos que se otorgan a tal condición. Revisar las estrategias de sobrevivencia en el sistema educativo permite reconocer al individuo como actor que reflexiona y decide en función de sus intereses. La propuesta de Dubet es asumir que si toda acción social responde a una acción racional, es posible identificar la manera en que cada actor se comporta como estratega que apunta hacia sus propios objetivos (parafraseando a Weber).

\section{Las estrategias de sobrevivencia educativa}

Nombrar como estrategias de sobrevivencia educativa a las acciones que los egresados llevaron a cabo para mantenerse matriculados en el bachillerato tiene una explicación conceptual. Primero, porque la propia SEP cuenta con un indicador educativo llamado "Esperanza de vida escolar", que para el ciclo escolar 2020-2021 era de 13.9 años para los habitantes de la república mexicana (SEP, 2021: 63). Por lo anterior, se retoma el lenguaje empleado por la propia institución educativa y se propone pensar que, si hay personas a las que se les asigna una "esperanza de vida escolar", también hay quienes desarrollan estrategias de sobrevivencia con la intención de prolongarla. El concepto también evoca las estrategias de sobrevivencia de las familias pobres que Larissa Lomnitz estudió en los años sesenta en la Ciudad de México (1998). Ese concepto le permitió explicar cómo grupos familiares migraban desde contextos rurales para establecerse en la dinámica de la ciudad e incorporarse a su economía industrial. Ella observó que esas familias recurrían al desarrollo de redes de apoyo mutuo para sobreponerse a la carencia de empleo, seguridad social y contingencias económicas, entre otras cosas. Estos recursos se empleaban como estrategias de sobrevivencia que les permitían sobreponerse a los cambios estructurales, pero también a los intergeneracionales, donde algunas de las cosas que parecían estar dadas se transformaban.

Al analizar los relatos biográficos y antecedentes familiares de los egresados con los que se trabajó en esta investigación, se asumió que no se estaba ante jóvenes que provenían de familias privilegiadas, ni tampoco ante sujetos que contaron con todos los recursos materiales e inmateriales para mantenerse enfocados en sus estudios. Por lo tanto, la recuperación de sus estrategias de sobrevivencia educativa permite indagar en la capacidad que desarrollan los estudiantes para mantenerse en el sistema educativo cuando no cuentan con los recursos económicos para hacerlo o cuando su estructura familiar encuentra problemas para convertir a lo educativo en parte central de sus actividades cotidianas. Lo que surge de estas experiencias es la posibilidad de comprender las construcciones individuales y colectivas que los estudiantes elaboran a partir de la conciencia que tienen del funcionamiento de la institución educativa para permanecer y moverse a través de ella.

Para ilustrar los resultados que se obtuvieron del análisis de las trayectorias a los egresados, se presentan algunos relatos con los que se elabora una tipología de las estrategias de sobre- 
vivencia educativa, identificadas al analizar sus relatos; en ellas es posible observar diferencias en cuanto a los hechos que las motivan, pero también, permiten entrever cómo los egresados apelaron a su capacidad de agencia para sobreponerse y mantenerse matriculados en el bachillerato tecnológico.

\section{La Comunicadora y las becas como estrategia de sobrevivencia}

La Comunicadora, además de haber estudiado en el CECyTEJ, cursó la licenciatura en Ciencias y Técnicas de la Educación en Guadalajara. Su interés por cursar la educación superior estuvo marcado por el hecho de que ella no quería trabajar en la industria, lo que puede verse como una forma de resistencia al destino laboral que pareciera presentarse en el entorno, ya que la industria aparece como el principal mercado de trabajo en El Salto.

Sus padres contaban con estudios de nivel básico, formaron una familia de siete personas (los padres y 5 hijos). Ella relata que para estudiar el bachillerato no contó con el apoyo de su padre, ya que desde su punto de vista las mujeres no necesitaban de los estudios a lo largo de sus vidas.

...para mi papá, la mujer no tiene porqué estudiar si la van a mantener. Mi mamá todo lo contrario, "si tú quieres estudiar, estudia, yo te apoyo y lo hacemos". Pero mi papá fue el que no, no le gustaba mucho la idea de que una mujer estudiara, mis hermanos sí pero nosotras no (Ia Comunicadora, egresada en 2007, con estudios de licenciatura, padre policía con secundaria).

Para sobreponerse a esa situación, comenzó a trabajar en algunos negocios cercanos a su hogar y después en algunos lugares que le permitían compaginar los estudios.

Mi primer trabajo fue en una papelería cerca de mi casa, trabajé ahí como un año, de ahí cubría obviamente gastos de la escuela y en cuestión de papelería, porque me ayudaban también aparte de un sueldo, que pues obviamente no era mucho pero te ayuda ¿A qué edad fue el trabajo de la papelería? Acababa de salir de secundaría, tenía 15 (Ia Comunicadora, egresada en 2007).

Estudiar y trabajar fue una decisión que racionalizó y que la llevó a cabo apelando a su capacidad de actoría, con la que pudo desplegar sus propios esfuerzos. Sin embargo, en su relato también se hizo evidente que, tanto para ella como para otros egresados, fue necesario recurrir a apoyos institucionales a fin de continuar estudiando, lo cual se presenta como una estrategia de sobrevivencia educativa.

Los apoyos institucionales se presentan a manera de becas, las cuales se entregan a los estudiantes que tienen problemas económicos para seguir estudiando. Las becas son un recurso que puede ofrecer la misma escuela o que los propios estudiantes pueden gestionar en otras instancias. 
¿Recibiste algún tipo de apoyo o beca? Sí, de transporte por mi promedio y por alimentos también ¿En qué consistía la de transporte? Me depositaban al mes una cantidad, te voy a decir como mil y fracción, dos mil pesos a lo mucho para ayuda de transporte. Era como una ayuda de transporte y la mantenías tú. ¿Sabes también cual tuve? La de colegiatura. Varios semestres no pagué colegiatura porque tenía buen promedio (la Comunicadora, egresada en 2007).

Entre los egresados se identificó que 13 de los 16 entrevistados contaron con algún tipo de beca durante el bachillerato y que esos recursos contribuyeron a que siguieran estudiando y eventualmente egresaran.

Tabla 2. Becas a las que accedieron los egresados

\begin{tabular}{|c|c|c|}
\hline Nombre de la beca & Tipo & Proveedor \\
\hline Programa Oportunidades & Económica & Gobierno Federal \\
\hline De transporte & En especie & Gobierno Estatal \\
\hline De colegiatura & Económica & CECyTEJ \\
\hline De alimentos & En especie & CECYTEJ \\
\hline
\end{tabular}

Fuente: Elaboración propia.

Los programas de becas son un recurso que despliega el sistema educativo en busca de disminuir el abandono escolar y de mejorar el rendimiento académico. En México existen diferentes estudios que pretenden identificar el impacto que tienen los programas de becas en el rendimiento escolar, donde se puede observar que existe una especie de tendencia positiva, aunque no tan significativa, entre el rendimiento escolar y los estudiantes que reciben una beca (Álvarez et al., 2012; Martínez, 2013; González, 2006).

En esos trabajos se identifica que los programas de becas han tenido mejores resultados al tratar de fomentar la permanencia de los estudiantes en la escuela. Los egresados de CECYTEJ pudieron acceder a esas becas de manera simultánea, lo que permite comprender que fue a través de éstas que algunos de los egresados continuaron estudiando.

Las becas son un recurso que los sujetos incorporan a su historia educativa. Una forma de capital cultural (Bourdieu, 2001) al que acceden debido al conocimiento que tienen del entorno y la manera en que aprenden a moverse en lo institucional. Esta aseveración se sustenta en el hecho de que no cualquier estudiante cuenta con el conocimiento de los recursos institucionales que le permitan acceder a ese tipo de programas. Por lo tanto, los egresados accedieron a esos recursos como una estrategia de sobrevivencia al entorno educativo, lo que les permitió mantenerse en la escuela y sobreponerse a contingencias económicas que experimentaron durante el bachillerato, principalmente a las derivadas de la falta de apoyo económico proveniente de sus familias de origen. 
Wacquant (2007) señala que las becas escolares constituyen una especie de respuesta a la desigualdad que se genera en las sociedades modernas con el retiro del estado de bienestar. Esta situación se propició debido a que el Estado ya no pudo garantizar que todos los niños y jóvenes acudieran a la escuela, ni tampoco que contaran con los recursos necesarios para hacerlo.

Las becas entonces forman parte de los recursos que las instituciones (principalmente de gobierno) ponen a disposición de los sectores sociales más necesitados para mantenerse en el sistema educativo, bajo el supuesto de que esto les permitirá incrementar la probabilidad de mejorar sus condiciones de vida (De la Rocha, 2006).

\section{El Federal y el bachillerato tecnológico como estrategia para ingresar a otros campos}

Otro caso que ilustra la manera en que los egresados despliegan estrategias que les permiten concretar sus proyectos educativos es el del Federal, quien poco antes de egresar del CECyTEJ se convirtió en padre de familia.

El Federal es uno de los egresados que mayor contacto tuvo con la industria antes, durante y después del bachillerato, ya que comenzó a trabajar en ella a los 16 años como obrero. Vincular lo laboral con sus estudios lo llevó a interrumpir el bachillerato en dos ocasiones.

¿Cómo fue que ingresaste a CECyTEJ? Estuve ahí en tres periodos. Entré por examen la primera vez y salí, no acabé el primer semestre, así fue también en la segunda ocasión, también pasó lo mismo; hice el examen de admisión, entré y salí y no duré tampoco el primer semestre porque ya trabajaba y estudiaba... y se me hacía muy pesado. ¿Dónde trabajabas? Estaba trabajando en Sanmina, aquí en la de carretera a Chapala y Carretera al Castillo ¿Y qué edad tenías? 16 años (El Federal, egresado en 2006. Padre, albañil con estudios de educación primaria).

Una vez egresado y ya como padre de familia, se matriculó en la Universidad Tecnológica de Jalisco (UTJ), en la carrera de ingeniería en electromecánica. Eligió esa carrera porque era el campo de conocimiento con el que se sentía identificado, aunque en realidad su interés principal estaba en convertirse en policía, algo que siempre había deseado.

Su ingreso a UTJ estuvo influenciado por uno de sus amigos que laboraba en la Policía Federal y que le comentó que las condiciones de trabajo eran mejores para aquellos que contaban con un título universitario. El Federal desarrolló una estrategia de sobrevivencia educativa que postriormente le permitiría incorporarse a un mercado laboral fuera de la industria, sin tener que renunciar a la afinidad que para entonces ya tenía con ésta. Al contar con estudios de bachillerato pudo matricularse en una carrera para incursionar en una mejor posición en la Policía Federal.

Esto se presenta como una doble estrategia; una para enriquecer su perfil curricular y otra para trasladarse a un campo laboral distinto al que ya había experimentado. 
Yo quería estudiar algo que a mí me gustara para poder entrar a la policía, que yo sé que puedes entrar hasta con la secundaria pero sí tienes mejores posibilidades si vas más preparado. La otra razón fue que si yo no quedaba en la policía, [así] podía tener herramientas con las cuales yo poder trabajar aquí en la industria en el sector privado y poder desarrollarme como tal (El Federal, egresado en 2006).

El Federal también hizo cálculos sobre las posibilidades que le brindaba su entorno inmediato y sobre éstas es que estudió una carrera relacionada con el mercado de trabajo de la industria, pero que al terminarla eligió una opción fuera de él.

\section{María y las redes de apoyo familiar}

Otra de las formas en que se presentan las estrategias de sobrevivencia educativa está en la construcción de redes de apoyo familiar, las cuales son empleadas para conseguir apoyo simbólico y económico entre los egresados que provienen de familias donde la economía del hogar no abona a los proyectos educativos de los hijos. Estas redes se constituyen entre distintos sujetos con diferentes jerarquías de parentesco, entre las que destaca el apoyo de las madres a sus hijas/os, entre hermanas, o el apoyo de tías, primos, padrinos, entre otros.

Una egresada que permitió profundizar en este tipo de estrategias fue María, quien al momento de la entrevista trabajaba y esperaba continuar estudiando en un futuro. Ella comentó que esta situación formaba parte de un proyecto que había elaborado con una de sus hermanas que también egresó del CECyTEJ, ya que su familia (padre obrero, madre ama de casa) no contaba con los recursos económicos para que ellas siguieran estudiando, por lo que acordó con su hermana trabajar y ayudarle con el pago de su colegiatura para que, una vez egresada, ella hiciera lo mismo con María.

¿Cómo es que le ayudas a tu hermana? Bueno, hizo varias veces intentos a la UdeG y no quedó, entonces tuvo que hacer trámites a una universidad de paga. Entonces ahorita la estoy apoyando económicamente en ese aspecto ¿Qué estudia ella? Administración ¿En qué semestre va? Ya es su último semestre (María, egresada en 2016. Padre obrero con estudios de secundaria).

En el caso de María se observa cómo las relaciones de intercambio al interior de la familia o del círculo social inmediato permiten a algunas egresadas sobreponerse a contingencias económicas y familiares, incluso a las restricciones que por su condición de género han encontrado en sus vidas. Se trata de procesos de intercambio que en su momento observó Lomnitz (1998) como recursos que permiten a los sujetos suplir la ausencia de prestaciones sociales en familias pobres.

Investigadores como Enríquez (2014), Lomnitz (1998), González (1997, 2006), Solís, Blanco y Robles (2015), han llevado a cabo diferentes investigaciones que permiten comprender la manera en que las familias de escasos recursos económicos se sobreponen a la pobreza y 
a la vulnerabilidad en contextos urbanos, destacando cómo ese tipo de familias desarrollan una marcada capacidad de adaptación a las crisis económicas a través de la construcción de redes de intercambio y de su incorporación a actividades productivas alternativas de autoabasto (González, 2006: 245).

Larissa Lomnitz fue pionera en este tipo de trabajos al estudiar las formas de socialización que se presentaban en algunas barriadas de la Ciudad de México en los años sesenta. En su trabajo sugirió pensar en las repercusiones que tienen en las familias más vulnerables las estructuras sociales que dificultan sus posibilidades de incorporarse a la economía industrial. Una economía que centraliza la producción económica en las grandes ciudades y orilla a los sujetos a migrar a lugares donde carecen de la preparación, así como de los recursos económicos y sociales que les permitan incorporarse a la actividad económica.

Sobre este supuesto es que Lomnitz se pregunta ¿Cómo sobreviven los que quedan marginados de la economía industrial? Una de las respuestas que encuentra en su análisis es que en estos grupos sociales se desarrollan comunidades basadas en redes de intercambio de favores y redes de apoyo mutuo, lo que les permite sobrevivir a las crisis económicas estructurales y a las crisis económicas internas de los hogares.

Lomnitz (1998: 26) también observa que esas redes de intercambio se presentan con diferente intensidad, lo cual está determinado por el tipo de parentesco y el peso que se da a la vecindad. Por lo que esas redes terminan por suplir con ayuda mutua la falta de seguridad social que implica quedar al margen de la economía industrial.

Al analizar las trayectorias de los egresados a partir del concepto de estrategia de sobrevivencia, se comprendió que ellos también las han implementado para sobreponerse a las condiciones de constreñimiento institucional del entorno. Las estrategias de sobrevivencia permitieron a 11 de los 16 egresados entrevistados incorporarse a instituciones de educación superior fuera de su comunidad.

El caso de María permite observar cómo el recurso de intercambio familiar contribuye a que ella y a su hermana desarrollen una estrategia para seguir estudiando. Ese intercambio implica un esfuerzo importante que está dispuesta a hacer, primero, por la intensidad del parentesco que tiene con la persona con quien establece la red de intercambio, pero también por el hecho de asumir que en ese acuerdo existe la posibilidad de monetizar los títulos escolares que puedan adquirir en el entorno inmediato en el que se encuentran.

¿Y tus intereses principales, cuáles son? Ahorita, mis intereses... mi hermana que está estudiando, personalmente me he hecho el propósito de apoyarle (María, egresada en 2016).

\section{Alan y la vinculación de la educación con el trabajo a edad temprana}

Alan es un egresado que proviene de una familia monoparental con jefatura femenina. Así como él, en la investigación se registraron los relatos de otros tres egresados con el mismo perfil fa- 
miliar. En comparación con los egresados que provienen de familias nucleares, estos egresados no siempre tienen que lidiar con la renuencia de la figura paterna a que sigan estudiando. Sin embargo, sus madres se enfocaron en trabajar para mantener sus hogares, propiciando que lo educativo tuviera menos seguimiento.

Aun así, en tres de los cuatro casos se identificó en que los egresados estaban matriculados en una institución de educación superior al momento de la entrevista y que una más (Gaby) laboraba en una empresa de transporte en un puesto directivo, por lo que en sus casos la estructura familiar no parece condicionar lo educativo de forma significativa.

Mercedes González de la Rocha (1997) realizó un estudio sobre familias con jefatura femenina en Jalisco, en el que abona a lo encontrado por Lomnitz (1998). Ella identificó que en este tipo de familias pueden encontrarse en una situación de mayor vulnerabilidad social cuando se aíslan, pero cuando desarrollan redes de apoyo e intercambio se fortalecen.

Esta autora observó a dos familias con esas características, en las que identificó que los hijos no necesariamente dejaron de estudiar al incorporarse de manera temprana al mercado de trabajo. Viendo a esta incorporación más como una forma de sobrevivencia que como un problema. En esas familias observa que existe un proyecto para monetizar la educación a mediano y largo plazo. En sus hallazgos también destacó que en este tipo de hogares no se obligaba a los hijos a dejar la escuela por lo laboral.

Uno de los aspectos que González puntualiza es que en algunos casos los estudiantes provenientes de este tipo de familias llegan a vincularse en actividades curriculares a las que no siempre pueden incursionar cuando la jefatura está a cargo de la figura masculina. Por ejemplo, identifica que los hijos de un hogar con esta estructura compaginan los estudios con el trabajo, pero también con la incursión en compañías de teatro o coros musicales, por lo que asume que en estos hogares existe la posibilidad de encontrar mayor democracia que en familias de los mismos estratos pero con jefatura masculina.

Aún y cuando los egresados con este perfil parecían estar consolidando sus trayectorias educativas, se observó que encontraron algunas contingencias para mantenerse matriculados en diversos momentos de su historia. El caso de Alan permite comprender que los jóvenes con este perfil también apelan tanto a redes de apoyo familiar como a vincular lo educativo con lo laboral, pues él relató que su trayectoria laboral comenzó a los nueve años y que lo orilló a transitar por diversos empleos. Alan comentó que mientras estudiaba en el CECyTEJ contó con una beca económica y otra para recibir alimentos en el plantel. Relató que con esos recursos y los empleos que tuvo en su paso por el CECyTEJ pudo hacerse cargo de sus propios gastos.

Es un orgullo saber que desde la secundaria me sufrago todos mis gastos estudiantiles. Desde la secundaria tenía un puestecillo de raspados o lo que sea, vendía donas en la madrugada, y me gustaba, y yo sacaba para pagar mis gastos, apoyando a mi familia obviamente. En la prepa igual, trabajaba y estudia- 
ba, y actualmente en la universidad también, digo, es algo que me llama la atención y se me hace chido (Alan, egresado en 2013. Madre estilista con estudios de secundaria).

Si se considera que algunas investigaciones sobre trayectos educativos han identificado que los estudiantes provenientes de familias monoparentales están más expuestos a abandonar o interrumpir sus estudios que los estudiantes provenientes de familias nucleares (Blanco, Solís, Robles, 2015), se puede asumir que en este grupo de egresados del CECyTEJ esto no necesariamente se presenta de esa manera.

Tampoco se presenta como una regla que cuando un estudiante trabaja verá truncada su trayectoria educativa. La vinculación del trabajo con los estudios se presentó en los relatos de los egresados como una decisión que tomaron para hacerse cargo desde temprana edad de sus gastos personales, pero también de sus destinos escolares. En los casos que se registraron en esta investigación esa decisión arrojó resultados positivos, aunque queda pendiente conocer (en otro posible estudio), qué tanto influyó lo laboral y la falta de apoyo familiar en el abandono de los no egresados.

\section{Lo que permite comprender las estrategias de sobrevivencia educativa}

Tabla 3. Las estrategias de sobrevivencia educativa de los egresados

\begin{tabular}{|c|c|}
\hline Estrategia & Recurso empleado \\
\hline Gestionando becas & $\begin{array}{c}\text { Económicas } \\
\text { De condonación de gastos escolares }\end{array}$ \\
\hline $\begin{array}{c}\text { Inscribiéndose al bachillerato } \\
\text { tecnológico }\end{array}$ & $\begin{array}{r}\text { Para no interrumpir sus estudios y preparar su incursión tanto } \\
\text { al mundo laboral como a la vida adulta }\end{array}$ \\
\hline $\begin{array}{c}\text { Estableciendo redes de apoyo e } \\
\text { intercambio familiar }\end{array}$ & $\begin{array}{c}\text { Con hermanas } \\
\text { Con la figura materna }\end{array}$ \\
\hline $\begin{array}{c}\text { Vinculando lo educativo } \\
\text { con lo laboral }\end{array}$ & En empleos eventuales, de medio tiempo \\
\hline
\end{tabular}

Fuente: elaboración propia.

Los relatos de los egresados permiten identificar algunas estrategias de sobrevivencia: la primera se presenta al gestionar becas de distintos tipos en forma simultánea, así como también la incorporación al bachillerato tecnológico termina por ser una estrategia que los habilita para seguir estudiando en un contexto en el que las condiciones para que los jóvenes estudien son limitadas. Asimismo, se identificó que algunos jóvenes establecen redes de apoyo e intercambio familiar y que otros deciden vincular lo educativo con lo laboral para seguir estudiando.

Estas estrategias de sobrevivencia son algunas de las que alcanzaron a observarse durante la elaboración de este trabajo, pero es pertinente señalar que no son las únicas pues existen 
otras que producen los estudiantes que pertenecen a este y a otros niveles educativos, las cuales están estrechamente relacionadas con el origen familiar y el contexto en el que los jóvenes se encuentran. Por ejemplo, se pueden encontrar estudiantes de educación superior que recurren a becas de manutención para seguir estudiando, ya sea en licenciaturas o posgrados. Mientras que los que acuden a escuelas particulares tienen la posibilidad de adscribirse a programas de financiamiento, ayudantías y descuentos institucionales para matricularse en el programa en el que están interesados. También vale la pena pensar que en el periodo en el que se lleva a cabo este trabajo se ha implementado una política federal para ofrecer becas a los estudiantes de todos los niveles de educación pública que lo soliciten, conocidas como "Becas Benito Juárez", que han despertado diferentes debates en la esfera pública y que valdría la pena darles seguimiento a través de estudios longitudinales, para evaluar el impacto que tienen en la disminución del abandono escolar, principalmente en las regiones y niveles educativos donde la SEP ha identificado que se presenta con mayor persistencia.

Por otro lado, es pertinente pensar que, en el contexto de la pandemia de Covid-19, se abre la posibilidad de identificar las estrategias que los estudiantes han empleado para no interrumpir sus actividades educativas, aún cuando las escuelas suspendieron sus actividades presenciales adscribiéndose a las dinámicas de educación a distancia, lo cual implica retos para sus economías y estructuras familiares. Las estrategias que los estudiantes de diferentes niveles han empleado en este periodo extraordinario para la humanidad posibilitan conocer sobre las redes de apoyo que constituyeron con otras personas, así como las acciones que llevaron a cabo para mantenerse matriculados a pesar de que en sus contextos inmediatos experimentaron contingencias.

Indagar en las experiencias que constituyen los estudiantes permite conocer a mayor profundidad sus trayectorias y poner énfasis en los sucesos importantes que las integran, ya que cada estudiante o egresado representa la posibilidad de conocer una o más estrategias de sobrevivencia educativa que pueden ser empleadas de manera simultánea para egresar de un nivel educativo. Con los resultados de este tipo de estudios se incrementa la posibilidad de producir conocimiento sobre el sujeto colectivo que constituyen los estudiantes.

También vale la pena pensar que, así como el conocimiento de las causas que detonan el abandono escolar en bachillerato ha servido para atender esa problemática, conocer las estrategias y los recursos que los egresados emplean para permanecer en las instituciones educativas puede contribuir a mejorar algunas de las estrategias que las instituciones educativas y el Estado implementan para retener a los estudiantes que están en riesgo de abandono.

\section{Algunas reflexiones finales}

Este trabajo puede contribuir a reflexionar sobre las diferentes problemáticas que experimentan tanto los estudiantes que cuentan con recursos limitados para desenvolverse académicamen- 
te, como aquellos que se encuentran en comunidades extrametropolitanas donde la oferta en educación media y superior es limitada y en ocasiones nula, debido a la insuficiente construcción de planteles, dando prioridad a los municipios de la zona metropolitana de Guadalajara (capital del estado) y restringe las aspiraciones de los jóvenes que tienen la intención de seguir estudiando en los municipios más alejados.

Otra problemática que se identificó en esta investigación y que experimentaron de manera exclusiva las egresadas con las que se dialogó, es la falta de apoyo que reciben de la figura paterna para estudiar el bachillerato. Este es un fenómeno que probablemente se presenta en otros municipios y responde a una lógica intergeneracional, consecuencia de la potestad que tienen los varones jefes de familia sobre los destinos escolares de sus hijas, que en casos como los que aquí se presentan terminan por restringir sus posibilidades de estudiar en niveles superiores e incorporarse a mercados de trabajo afines a sus intereses.

El seguimiento que se hizo a las trayectorias de las egresadas que experimentaron esta problemática permitió observar que, tanto el egreso del bachillerato como la adquisición del capital cultural que provee la escuela a manera de certificaciones, funcionan como variable que contribuye a que las egresadas obtengan un mayor control en su incursión a la vida adulta, algo que también abona al rompimiento que las mujeres demandan con el pacto patriarcal.

Por otro lado, las egresadas y los egresados con los que se dialogó hicieron evidente que las redes de apoyo familiar que Lomnitz (1994) identificó como mecanismo de resistencia y acompañamiento comunitario hace varias décadas siguen vigentes, y que tanto las mujeres a las que se les niega el apoyo para estudiar como a los varones que provienen de familias monoparentales les resulta indispensable contar con este tipo de apoyos, pues les permiten acceder a recursos simbólicos y materiales con los que pueden seguir estudiando.

También se observó que los estudiantes que trabajan confirmaron lo complicado que es vincular ambas actividades, más aún cuando se trata de empleos en la industria, mercado de trabajo que casi no distingue entre trabajadores de tiempo completo y estudiantes que requieren empleos con jornadas acotadas. Por lo general, los egresados que trabajaron lo hicieron en jornadas completas, en puestos de baja jerarquía que mermaron su rendimiento escolar, aunque con todo y eso evidenciaron que trabajar y estudiar también se traduce en una estrategia de sobrevivencia educativa, pues en algunos casos la experiencia laboral les ayuda a vincularse con otros campos de conocimiento, situación que contribuye a que generen nuevas aspiraciones y los faculta para contar con recursos para seguir estudiando:

¿Cómo le hacías para estudiar y trabajar? De hecho, tenía permiso para llegar a las ocho (de la mañana), perdía una hora de clase... ¿llegabas en vivo? Y llegaba corriendo ¿Y qué tal era eso de llegar sin dormir? Muy pesado. La verdad es que pues todavía las primeras clases de la mañana sí estaba como que muy atenta, pero ya lo que eran las últimas dos horas era cuando ya... era muy difícil. Llegaba un momento 
en el que a veces pedía permiso a los maestros para retirarme un momento al baño, iba y me mojaba la cara, porque ya, yo sentía que el sueño me estaba ganando ( $L a$ Doña, comenzó a trabajar a los 15 años, padre abogado).

Al analizar de manera colectiva los relatos de los egresados se identificó que ellos convirtieron el bachillerato tecnológico en una estrategia de sobrevivencia educativa, pues casi todos dijeron haber buscado un espacio en la preparatoria regional de la UdeG que se ubica en El Salto, pero que fueron rechazados, razón por la que acudieron al CECyTEJ y encontraron mejores condiciones para seguir estudiando. Por lo tanto, matricularse en una institución de educación tecnológica de la que se habla menos a nivel local, se convirtió en un recurso con el que pudieron seguir estudiando.

¿Para ti cuál era la fortaleza de CECyTEJ? Que cualquier persona puede entrar, cualquiera. No era nada de que "refrenda" o que "espérate, estamos llenos", yo no supe de nadie que no pudiera entrar, todos entraban (El Freelance, egresado en 2007. Padre policía con estudios de secundaria).

Con la información que se recogió en esta investigación se pudo comprender que las trayectorias de este grupo de egresados se constituyen en un entorno constreñido por una oferta educativa limitada, así como por los factores socioculturales que forman parte de sus experiencias escolares. También se considera constreñido pues carece de mecanismos para incorporar y retener a los estudiantes que ocupan los espacios con los que se cuenta en el bachillerato.

Los relatos de los egresados de bachillerato tecnológico permitieron comprender los significados que producen sobre la institución educativa a la que se adscriben, pues ésta los afilia y les permite protagonizar una de sus primeras producciones culturales como colectivo ya que se observó que, al egresar, la mayoría de ellos se convirtieron en el integrante de sus familias que mayor escolaridad ha alcanzado (11 de 16 casos), por lo que protagonizan un fenómeno de movilidad escolar intergeneracional que los faculta para adscribirse a otros niveles educativos, e incluso aspirar a otros campos laborales. Al respecto, se observó que 11 de los 16 egresados con los que se dialogó han estudiado en algún momento en instituciones de educación superior (principalmente en programas de ingeniería, dos de ellos ya egresados), lo que confirma que, a través de estas estrategias, los sujetos adquieren un mayor control sobre sus experiencias escolares y sobre la orientación de los estudios que realizan, condición que eventualmente les permite integrarse a diferentes campos laborales, ya que se pudo identificar que, al momento de la entrevista, los egresados habían incursionado en diferentes empleos, la mayoría de ellos considerados como estables, pues se ubican en empresas que les ofrecen prestaciones de ley, como es el seguro social y créditos para vivienda. Condiciones que para ellos resultan significativas y los motivaban a ampliar su trayectoria escolar para competir por otros puestos. 
Estrategias de sobrevivencia educativa de los egresados de bachillerato tecnológico de CECyTEJ, El Salto

Tabla 4. Ocupación de los egresados cuando se llevaron a cabo las entrevistas

\begin{tabular}{|l|l|}
\hline \multicolumn{1}{|c|}{ Egresados } & \multicolumn{1}{c|}{ Ocupación } \\
\hline El Federal, Socorro, Gaby & Encargados de área en alguna empresa \\
\hline $\begin{array}{l}\text { La Comunicadora, el Freelance, Grande, } \\
\text { Alan, María, la Deportista }\end{array}$ & Empleados en alguna empresa o institución \\
\hline La Doña, Santos, Larrión & Técnicos en empresas \\
\hline Manuel, Hilda, la Maestra & Estudiantes de educación superior \\
\hline Karla & Estaba embarazada y enfocada en el hogar \\
\hline
\end{tabular}

Fuente: elaboración propia, con datos de los egresados.

Por último, vale la pena mencionar que este trabajo se limita al análisis de los testimonios que fue posible recoger de los sujetos que estudiaron en el CECyTEJ, El Salto, que sí egresaron, pero queda pendiente para investigaciones futuras recuperar la voz de quienes no lo lograron, con el propósito de conocer cuáles fueron las situaciones que experimentaron tanto en sus contextos inmediatos como en la operación del plantel, para así identificar aquellos que no coadyuvaron a su continuidad como estudiantes matriculados. Conocer la voz de los no egresados es un pendiente que podría abonar al fortalecimiento de las políticas públicas que se implementan para disminuir la interrupción y el abandono escolar que se presentan en este nivel educativo a nivel nacional.

\section{Referencias}

Bertaux, D. (2005). Los relatos de la vida. Perspectiva etnosociológica. España: Ediciones Bellaterra. Blanco, E.; P. Solís (2015). Caminos desiguales: trayectorias educativas y laborales de los jóvenes en la Ciudad de México. México: COLMEX, INEE.

Boado, M. (2013). Movilidad ocupacional y mercado de trabajo: las caras ocultas del empleo urbano en Montevideo. Notas para una investigación. Revista de Ciencias Sociales, (12). http:// www.rau.edu.uy/fcs/soc/Publicaciones/Revista/Revista12/Boado.html

Bourdieu, P. (1998). La Distinción. Criterios y bases sociales del gusto. Madrid: Taurus.

Bourdieu, P. (2001). Poder, derecho y clases sociales. Bilbao: Ed. Desclee.

Bourdieu, P. (2004). Los herederos. Los estudiantes y la cultura. Argentina: Siglo XXI.

Casillas, P. (2013). La flexibilidad laboral en la industria de la electrónica en México. Contextualizaciones Latinoamericanas, 5(8).

CEREAL (2015). El precio de la flexibilidad: Experiencias de trabajadores en la industria de la electrónica en México. Sexto informe sobre las condiciones laborales en la industria de la electró- 
nica en México. Centro de Reflexión y Acción Laboral. https://goodelectronics.org/wpcontent/uploads/sites/3/2015/03/filesnamePaying20the20price20for20flexibility2020workers2720experiences20in20the20electronics20industry20in20Mexico Spanish.pdf

Delgado, C. (2017). El empleo femenino en la industria de la electrónica de la ZMG y precariedad laboral. Expresión Económica, (27).

Dubet, F. (2005). Los estudiantes. Revista de Investigación Educativa, (1). https://www.uv.mx/ cpue/num1/inves/completos/Dubet.pdf

Dubet, F. (2010). La sociología de la experiencia. Madrid: Editorial Complutense.

Enríquez, R. (2009). El crisol de la pobreza. Mujeres, subjetividades, emociones y redes sociales. México: ITESO.

Fernández, L. (1994). Instituciones educativas. Argentina: Paidós.

Gabayet, L. (2006). Atrapadas en la flexibilidad y la precariedad en el trabajo. Las obreras de la industria de la electrónica de la zona metropolitana de Guadalajara, 1988-2004. Desacatos, (21).

Giddens, A. (2011). La constitución de la sociedad. Bases para la teoría de la estructuración. Argentina: Amorrortu Editores.

Giorguli, S. (2002). Estructuras familiares y oportunidades escolares de los niños y niñas en México. Estudios Demográficos y Urbanos, 17(3), 523-545. https://estudiosdemograficosyurbanos.colmex.mx/index.php/edu/article/view/1149/1142

González, M. (1997). Hogares de jefatura femenina en México. Patrones y formas de vida. XX Congreso Internacional de la Sociedad de Estudios Latinoamericanos (LASA), Guadalajara, México. http://lasa.international.pitt.edu/LASA97/rocha.pdf

González, M. (2006). Procesos domésticos y vulnerabilidad. Perspectivas antropológicas de los hogares con Oportunidades. México: CIESAS.

Greenpeace (2012). Estudio de la contaminación en la cuenca del Río Santiago y la salud pública en la región. México: UCCS.

INEE (2018). Abandono escolar en bachillerato: una mirada a las raíces del fenómeno.

https://historico.mejoredu.gob.mx/abandono-escolar-en-bachillerato-una-mirada-a-las-raices-del-fenomeno/

McCulligh, C. (25 de septiembre de 2006). Respirar veneno en Juanacatlán y El Salto, Jalisco. La Jornada. http://www.jornada.unam.mx/2006/09/25/eco-c.html

Partida, R. (2002). Localización industrial en la cuenca Lerma-Chapala-Santiago: el caso del Corredor Industrial Jalisco. El Cotidiano s/n. http://hdl.handle.net/123456789/60357

Partida, R. (2004). Las fases del desarrollo de la industria maquiladora electrónica en Jalisco. El Cotidiano, 20, (126). México: UAM-Azcapotzalco.

Ramos, J. (2014). Representaciones sobre el trabajo y la industria electrónica en una empresa de servicios de manufactura electrónica de la Zona Metropolitana de Guadalajara, México. 1er Congreso Latinoamericano de Estudiantes de Posgrado en Ciencias Sociales. México: FLACSO. 
Regalado, J. (2015). Relación ciudad-naturaleza y sus impactos sobre la salud. La destrucción del cordón natural de la ciudad de Guadalajara. En Garibay, et al. Salud ambiental en la zona metropolitana de Guadalajara. Imaginando futuros diferentes. México: Universidad de Guadalajara, 21-53.

Remedi, E. (coord.) (2004). Instituciones educativas. Sujetos, historia e identidades. México: Plaza y Valdés.

Rodríguez, J.; M. Cota (julio de 2006). Desarrollo del Parque Industrial El Salto, Jalisco. Cuadernos PROLAM/USP, 5(2), 83-104.

Salas, M. (2010). Corredor Industrial: proceso del desarrollo.

http://www.paginasprodigy.com/iee elsalto/C Industrial.pdf

Saraví, G. (2016). Juventudes fragmentadas. Socialización, clase y cultura en la construcción de la desigualdad. México: FLACSO, CIESAS.

SEMS (2015). Movimiento contra el abandono escolar. Jornadas de trabajo 2015. http://www.sems.gob.mx/work/models/sems/Resource/11390/8/images/jornadas 2015.pdf

SNIE (2019). Principales cifras del sistema educativo nacional 2018-2019. México: SEP.

https://www.planeacion.sep.gob.mx/Doc/estadistica e indicadores/principales cifras/ principales cifras 20182019 bolsillo.pdf

Vargas, R. (2000). Trayectoria profesional de los ingenieros en la industria maquiladora electrónica: el caso de Sanyo Video Componentes. Revista Electrónica de Investigación Educativa, 2, 2-22. https://www.redalyc.org/articulo.oa?id=15502201

Wacquant, L. (2007). Los condenados de la ciudad. Gueto, periferias y Estado. Argentina: Siglo XXI Editores. 